\title{
ASSOCIATION BETWEEN HIGH RISK BEHAVIOUR AND ALCOHOL DEPENDENCE SYNDROME
}

\section{Chandra Sekhar A ${ }^{1}$, Eshwar Reddy Ravula ${ }^{2}$.}

${ }^{1}$ Assistant Professor in Psychiatry, Viswabharathi Medical College and Hospital, Kurnool, Andhra Pradesh.

${ }^{* 2}$ Consultant Psychiatry, Clears water Hyderabad, Telangana.

\section{ABSTRACT}

Background: Alcohol use has been shown to be associated with high risk behaviour. However, the exact nature of this association remains to be clarified. The role of personality constructs of sensation- seeking and impulsivity has been implicated in high risk behaviour. Most of the published literature has been on population from the developed countries but not from the developing countries like INDIA have been few.

Methods: 60 male patients admitted with an ICD 10 diagnosis of alcohol Dependence Syndrome were studied using both self-report and semi structured interview. The data was analyzed using an Event- analysis technique for the occurrence of high risk behaviour, namely Road traffic Accidents, Crime and Violence, Selfinjurious behaviour and risky Sexual behaviour. Personality variables were studied using the Sensation Seeking Scale, Form V, Indian Adaptation and Barratt's Impulsivity Scale, version 11. Data was analysed using SPSS.

Results: Out of the 60 patients studied $68.3 \%(n=47)$ of the sample were exposed to high risk behaviour. Analysis of the data found evidence that Severity of Dependence, Personality Constructs of Sensation Seeking and Impulsivity, and family history of dependence showed significant association with high risk behaviour.

Conclusion: Amongst the high-risk behaviours in alcoholics, risky sexual behaviour was the commonest followed by road traffic accidents. The significance of personality constructs of sensation seeking and impulsivity in high risk behaviour needs to be evaluated more routinely in patients with alcohol dependence syndrome, as intervention strategies need to be planned keeping these in mind to improve the effectiveness of treatment.

KEY WORDS: High Risk Behaviour, Sensation Seeking, Impulsivity.

Address for correspondence: Dr. Chandra Sekhar A, Assistant Professor in Psychiatry, Viswabharathi Medical College and Hospital, Kurnool, Andhra Pradesh.

E-Mail: csreddy81@gmail.com.

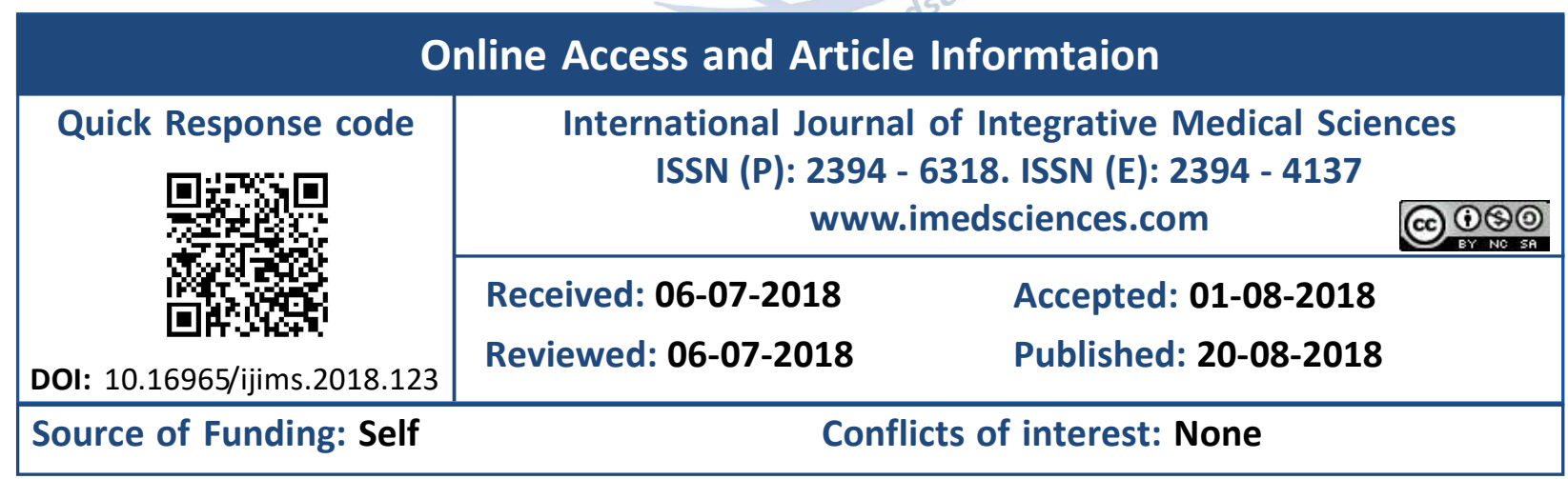

\section{BACKGROUND}

Alcohol is the most used drug in the world. It is mainly because alcohol is legal and socially acceptable in most countries [1]. Majority of people drink alcohol, generally for enjoyment, relaxation and sociability. Most people belongs to group of moderate drinkers and at this level, alcohol causes only a few adverse effects [2]. Actually, sensible drinking can even be beneficial for the health of adult [3]. Unlike the moderate drinking, experiencing of severe or frequent intoxication negatively affect short-term as well as long-term health condition. Often alcohol abuse and alcoholism does not affect just health of the drinker, but also harms drinker's family and friends, employer and the broader community. Even though, there are just a small proportion of people who consume alcohol at higher 
levels, the impact of this group on society is significant. The society suffers from heavy drinkers mentally as well as economically.

It is not just the treatment of alcohol related to illnesses problems such as cirrhosis of the liver, or cancer of the oesophagus or stomach, which is costly. Drinkers are frequently predisposed to common injuries. All this health related trouble together with often physical and mental incompetence caused by intoxication rise to decrease in productivity and to higher absence from work. Among others, alcohol abuse increases crimerelated costs as well as vehicle crash costs. Moreover, heavy drinkers and alcoholics are more likely to die prematurely (Harwood et al, 1998). Unfortunately, the burden of alcohol problems does not fall just on the abusers. It is primarily the rest of society, who bears these costs [4]. Alcohol Dependence Syndrome is one of the most common and one of the most researched illnesses among psychiatric disorders. The Global Burden of Disease Project estimated alcohol to be responsible for $1.5 \%$ of all deaths and $3.5 \%$ of the total Disability Adjusted Life Years (DALYs) [5]. In India, epidemiological studies have shown a prevalence rate of $16-50 \%$ for alcoholism [6]. The morbidity and mortality associated with alcoholism is further compounded by the concept of 'risk-taking'. Literature supports the notion that there are links between alcohol use and problematic or risky behavior; However, the nature of these links have often been explained in terms such as "involved," "implicated" and "associated" [7]. However, there is evidence from literature that drinking and drug use are associated with sensation seeking and risk-taking propensities, as measured by personality scales. Therefore, substance use and risky behavior might be part of a larger constellation of risk taking behavior $[8,9]$. Given the cultural differences in the patterns of substance use between the western society and developing countries, it is important to study the association of high-risk behavior with substance use in our country. This assumes greater significance in the background of rising incidence of high-risk behavior such as drunken driving related fatalities, increasing incidences of HIV, sexually transmitted diseases, crime and deliberate self-harm [10]. In this background, a study of the association between High Risk Behavior and Alcohol Dependence in a group of male alcohol dependent patients, being admitted to the in-patient facility of a Psychiatric unit would be beneficial in furthering our understanding on the subject.

\section{MATERIALS AND METHODS}

The study was conducted in the Department of Psychiatry at Narayana Medical College and Hospital in Nellore. The study was conducted on 60 male in-patients who were admitted in Hospital for the treatment of Alcohol related illness. The patients were screened to ensure that Male with ICD-10 diagnosis of Alcohol Dependence Syndrome in-patients. Male patients with any other co morbid Axis-1 psychiatric diagnosis, any other substance use, except tobacco and presence of cognitive impairment were excluded from the study. Consenting patients were then interviewed on Structured Clinical Interview for DSM-IV (SCID) to establish a diagnosis of alcohol dependence syndrome. SCID was also used to exclude any other co-morbid axis-I diagnosis. Patients were then screened using Clinical Institute Withdrawal Assessment for Alcohol (CIWA-AD) and Mini-Mental State Examination (MMSE) to ensure that patients were not in withdrawal state and had no cognitive impairment respectively. Patients who were deemed to be out of withdrawal state and not having any cognitive impairment were then assessed on the following by Severity of Alcohol Dependence Questionnaire (Sad-Q) [11], Measurement of Sensation seeking scale, form v: Indian adaptation [12], Measurement of impulsivity by Barratt's impulsiveness scale, version 11 ( bis-11) [13]. After the subjects are admitted to the wards, patients are screened for the presence of alcohol use and the presence of any major medical or psychiatric illness. Following this the data was collected in a systematic approach as presented in the flow chart on the following page. The order of presentation of various questionnaires and scales were maintained constant throughout the duration of the study.

Statistical analysis: The data was analyzed using a computerized Software Programme Statistical Package for the Social Sciences (SPSS 
version 21.0 for Windows). Descriptive statistics was used to describe the socio demographic variables. Independent Student t-test, ANOVAs was used to analyze the association between the various independent variables.

\section{RESULTS}

Fig. 1: Frequency of distribution of marital status.

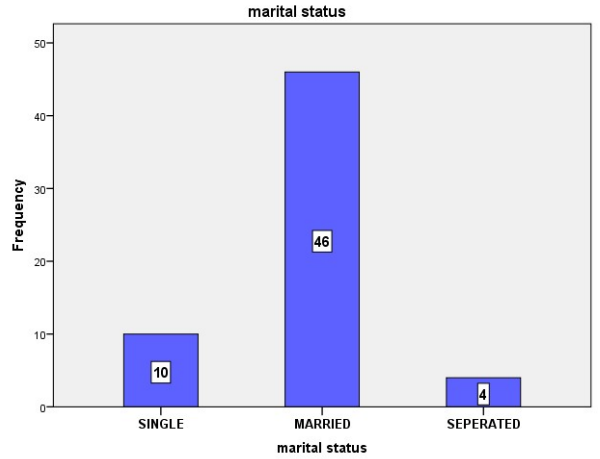

Frequency of distribution of marital status $(n=60): 76.7 \%$ of the total sample consisted of married men, $16.7 \%$ were single while $6.6 \%$ were separated

Fig. 2: Frequency of distribution of family type.

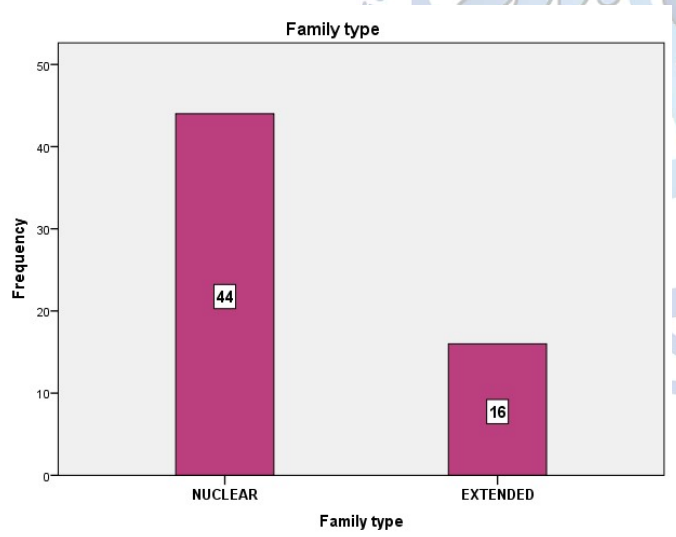

Frequency of distribution of family type $(n=60)$ : $73.3 \% \%$ of the total sample belonged to a nuclear family while $26.7 \%$ had an extended family.

Fig. 3: Frequency of distribution of education.

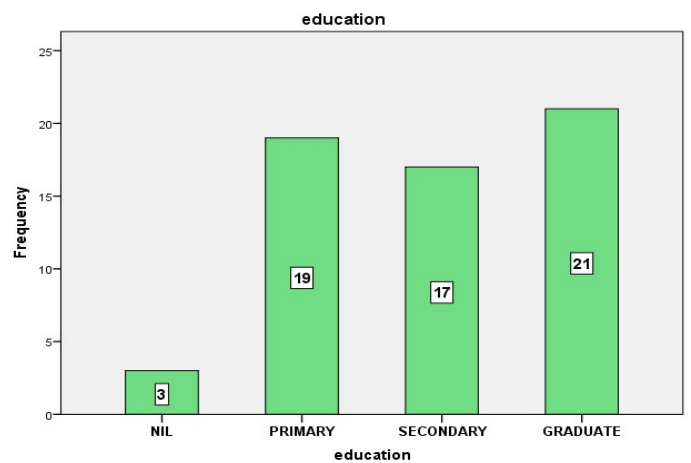

Majority of the sample (35\%) were graduates followed by individuals with primary education (31.7\%).
Fig. 4: Frequency of distribution of occupation.

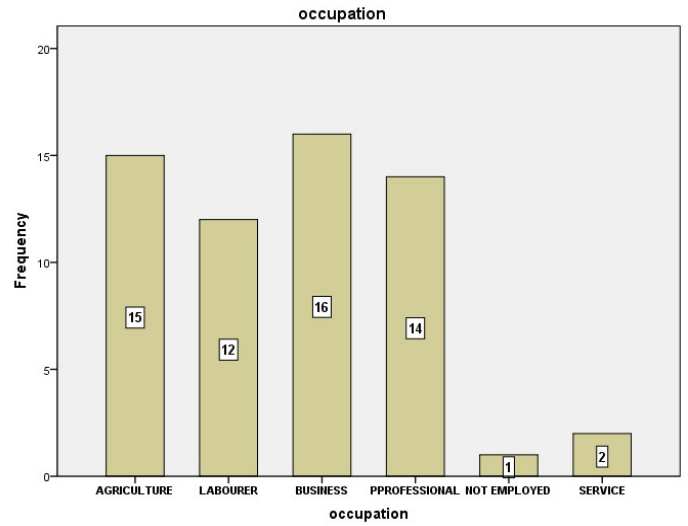

Occupation of $26.7 \%$ of the individuals who participated in this study was business closely followed by people with agriculture as the occupation (25\%) and $23 \%$ of the individuals were professionals.

Fig. 5: Frequency of distribution of family history.

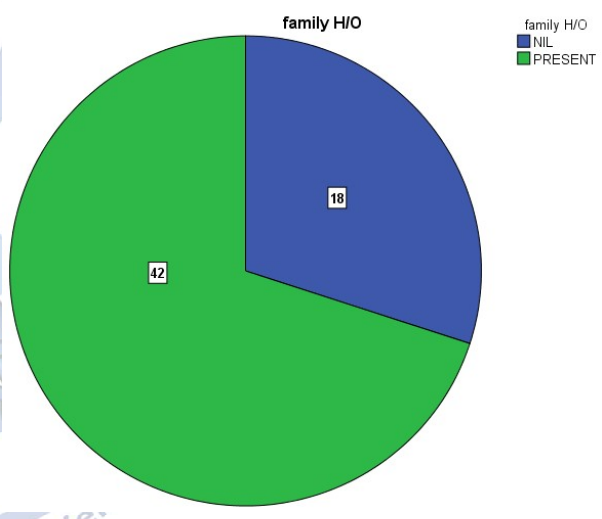

Note: $70 \%$ of the individuals had family history of dependence

Fig. 6: Frequency of distribution of severity of dependence.

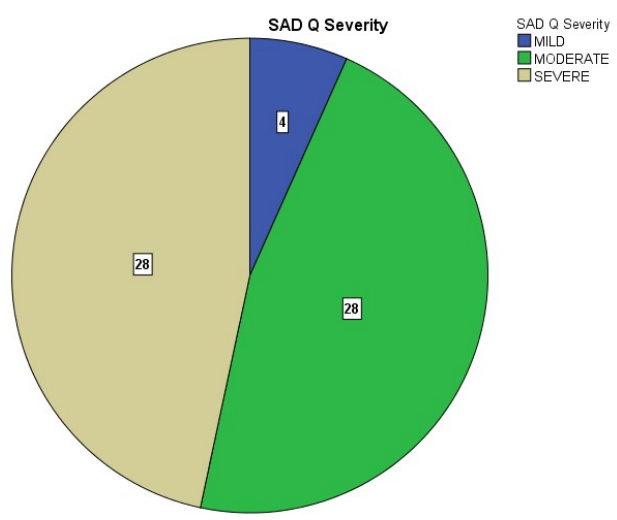

Note: Scores on SAD-Q reveal that $46.7 \%$ of the sample had severe dependence and the same percentage of the individuals had moderate dependence, while $6.7 \%$ of individuals had mild dependence. 
Fig. 7: Frequency of distribution of high risk behaviour.

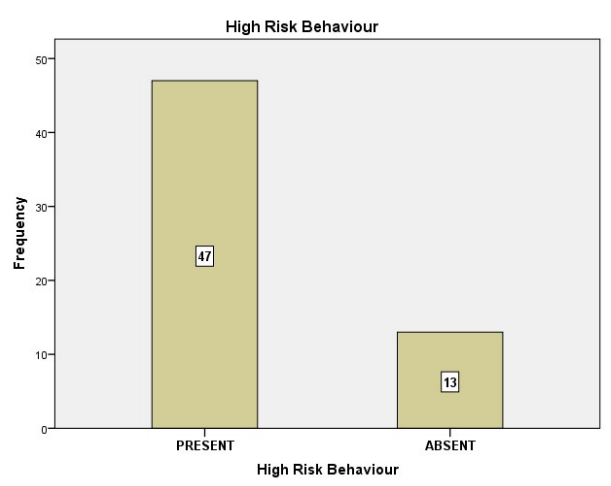

The analysis of the results showed that $68.3 \%$ $(n=47)$ of the sample had been involved in some form of High Risk Behaviour, with a temporal relationship with alcohol.

Table 1: Association between severity of dependence and High Risk Behaviour.

\begin{tabular}{|c|c|c|c|}
\hline Variables & SAD Q Severity & $\begin{array}{c}\text { Mean } \pm \text { SD/ Frequency } \\
(\%)\end{array}$ & $\begin{array}{c}\mathrm{t} \text {-value / Chi } \\
\text { square/ F-Value }\end{array}$ \\
\hline \multirow{3}{*}{ High Risk Behaviour } & MILD & $\begin{array}{l}\text { Present - 1 }(25.00 \%) \\
\text { Absent - 3(75.00\%) }\end{array}$ & \\
\hline & MODERATE & $\begin{array}{l}\text { Present - 19.(67.9\%) } \\
\text { Absent - } 9(32.1 \%)\end{array}$ & \\
\hline & SEVERE & $\begin{array}{c}\text { Present - } 27(96.4 \%) \\
\text { Absent }-1(3.6 \%)\end{array}$ & \\
\hline \multirow{3}{*}{ RTA } & MILD & $\begin{array}{l}\text { Present - } 0(0.00 \%) \\
\text { Absent - } 4(100.0 \%)\end{array}$ & \multirow{3}{*}{5.185} \\
\hline & MODERATE & $\begin{array}{l}\text { Present - } 15(53.6 \%) \\
\text { Absent - } 13(46.4 \%)\end{array}$ & \\
\hline & SEVERE & $\begin{array}{l}\text { Present - } 17(60.7 \%) \\
\text { Absent - } 11(39.3 \%)\end{array}$ & \\
\hline \multirow{3}{*}{ CRIME \& VIOLENCE } & MILD & $\begin{array}{c}\text { Present -0 }(0.0 \%) \\
\text { Absent - } 4(100.0 \%)\end{array}$ & \multirow{3}{*}{2.902} \\
\hline & MODERATE & $\begin{array}{l}\text { Present }-5(17.9 \%) \\
\text { Absent - } 23(82.1 \%)\end{array}$ & \\
\hline & SEVERE & $\begin{array}{l}\text { Present - } 9(32.1 \%) \\
\text { Absent - } 19(67.9 \%)\end{array}$ & \\
\hline \multirow{3}{*}{ SELF INJ. BEHAVIOUR } & MILD & $\begin{array}{l}\text { Present - 1 (25.0\%) } \\
\text { Absent - 3(75.0\%) }\end{array}$ & \multirow{3}{*}{2.502} \\
\hline & MODERATE & $\begin{array}{l}\text { Present - } 4(14.3 \%) \\
\text { Absent - } 24(85.7 \%)\end{array}$ & \\
\hline & SEVERE & $\begin{array}{l}\text { Present - } 9(32.1 \%) \\
\text { Absent - 19 (67.9\%) }\end{array}$ & \\
\hline \multirow{3}{*}{$\begin{array}{l}\text { HIGH RISK SEXUAL } \\
\text { BEHAVIOUR }\end{array}$} & MILD & $\begin{array}{l}\text { Present - 1 (25.0\%) } \\
\text { Absent - 3(75.0\%) }\end{array}$ & \multirow{3}{*}{$10.549 *$} \\
\hline & MODERATE & Present - $11(39.3 \%)$ & \\
\hline & SEVERE & $\begin{array}{l}\text { Present - } 22(78.6 \%) \\
\text { Absent - } 6(21.4 \%)\end{array}$ & \\
\hline
\end{tabular}

Level of significance: ${ }^{*}$-p $<0.05,{ }^{* *}$-p $<0.01,{ }^{* * *}$-p $<0.001$, NS-Not significant

High Risk Sexual Behaviour was found to be significantly associated with severity of dependence among the individuals.
Table 2: Association between severity of dependence and Sensation Seeking behaviour.

\begin{tabular}{|c|c|c|c|}
\hline Variables & SAD Q Severity & $\begin{array}{l}\text { Mean } \pm \mathrm{SD} / \\
\text { Frequency (\%) }\end{array}$ & $\begin{array}{l}\text { t-value / Chi } \\
\text { square/ F- } \\
\text { Value }\end{array}$ \\
\hline \multirow{3}{*}{ TAS } & MILD & $4.25 \pm 1.3$ & \multirow{3}{*}{$3.680^{*}$} \\
\hline & MODERATE & $4.79 \pm 0.8$ & \\
\hline & SEVERE & $5.21 \pm 0.7$ & \\
\hline \multirow{3}{*}{ ES } & MILD & $4.00 \pm 0.8$ & \multirow{3}{*}{0.842} \\
\hline & MODERATE & $3.93 \pm 0.9$ & \\
\hline & SEVERE & $4.18 \pm 0.5$ & \\
\hline \multirow{3}{*}{ BS } & MILD & $5.00 \pm 0.8$ & \multirow{3}{*}{1.988} \\
\hline & MODERATE & $5.07 \pm 0.9$ & \\
\hline & SEVERE & $5.46 \pm 0.7$ & \\
\hline \multirow{3}{*}{ DIS } & MILD & $4.50 \pm 1.0$ & \multirow{3}{*}{$5.199 *$} \\
\hline & MODERATE & $5.18 \pm 1.1$ & \\
\hline & SEVERE & $5.86 \pm 0.8$ & \\
\hline \multirow{3}{*}{$\begin{array}{l}\text { SENSATION SEEKING } \\
\text { TOTAL SCORE }\end{array}$} & MILD & $17.75 \pm 3.8$ & \multirow{3}{*}{$3.786^{*}$} \\
\hline & MODERATE & $18.96 \pm 3.2$ & \\
\hline & SEVERE & $20.71 \pm 2.2$ & \\
\hline
\end{tabular}

Level of significance: ${ }^{*}-p<0.05,{ }^{* *}{ }_{-}-p<0.01,{ }^{* * *}-p<0.001$, NS-Not significant

Severity of dependence was found to be significantly associated with disinhibition, thrill and adventure seeking behaviour.

Table 3: Association between severity of dependence and impulsive behaviour.

\begin{tabular}{|c|c|c|c|}
\hline Variables & SAD Q Severy & $\begin{array}{c}\text { Mean } \pm \text { SD/ } \\
\text { Frequency ( \% ) }\end{array}$ & $\begin{array}{c}\text { t-value / Chi } \\
\text { square / F-Value }\end{array}$ \\
\hline \multirow{3}{*}{ MOTOR } & MILD & $27.25 \pm 4.0$ & \multirow{3}{*}{$4.267^{*}$} \\
\hline & MODERATE & $28.82 \pm 3.2$ & \\
\hline & SEVERE & $30.75 \pm 2.6$ & \\
\hline \multirow{3}{*}{ NON- PLANNING } & MILD & $27.75 \pm 4.3$ & \multirow{3}{*}{$3.808^{*}$} \\
\hline & MODERATE & $29.43 \pm 3.2$ & \\
\hline & SEVERE & $31.07 \pm 2.1$ & \\
\hline \multirow{3}{*}{ cogNITIVE } & MILD & $21.50 \pm 3.3$ & \multirow{3}{*}{2.853} \\
\hline & MODERATE & $21.96 \pm 2.1$ & \\
\hline & SEVERE & $23.11 \pm 1.5$ & \\
\hline \multirow{3}{*}{ BIS-TOTAL SCORE } & MILD & $76.50 \pm 11.6$ & \multirow{3}{*}{$4.270^{*}$} \\
\hline & MODERATE & $80.21 \pm 8.1$ & \\
\hline & SEVERE & $84.93 \pm 5.5$ & \\
\hline
\end{tabular}

Level of significance: ${ }^{*}-p<0.05,{ }^{* *}-p<0.01,{ }^{* * *}$-p $<0.001$, NS-Not significant

Impulsive behaviour was found to be significantly influence by severity of dependence. Motor impulsivity and non-planning were much more in individuals with severe dependence when compared to that of individuals with moderate and mild dependence.

Table 4: Frequency of occurrence of HIGH RISK BEHAVIOUR in the sample $(n=60)$.

\begin{tabular}{|c|c|c|c|c|c|c|c|c|}
\hline \multirow{2}{*}{ TYPE } & \multicolumn{2}{|l|}{ Road Traffic Accidents } & \multicolumn{2}{|c|}{ Crime \&violence } & \multicolumn{2}{c|}{ Self Injurious Behaviour } & \multicolumn{2}{c|}{ Risky Sexual Behaviour } \\
\cline { 2 - 10 } & Present & Absent & Present & Absent & Present & Absent & Present & Absent \\
\hline FREQUENCY & 32 & 28 & 14 & 46 & 14 & 46 & 34 & 26 \\
\hline PERCENTAGE (\%) & 53.3 & 46.7 & 23.3 & 76.7 & 23.3 & 76.7 & 56.7 & 43.3 \\
\hline
\end{tabular}


On examining the frequencies of high risk behavior in the entire sample it was seen that the occurrence of risky sexual behaviour (56.7 $\%$ ) was the highest among the high risk behaviours, with $53.3 \%$ also being involved in road traffic accidents. Crime and violence was seen in $23.3 \%$ of the sample, whereas $23.3 \%$ showed self -injurious behaviour. These were values of occurrence of High Risk Behaviour without accounting for any temporal relation with alcohol use.

Table 5: Association between High Risk Behaviour and socio demographic variables.

\begin{tabular}{|c|c|c|c|c|}
\hline Variables & $\begin{array}{c}\text { High Risk } \\
\text { Behaviour }\end{array}$ & \multicolumn{2}{|c|}{ Mean \pm SD/Frequency (\%) } & $\begin{array}{c}\text { t-value/Chi } \\
\text { Square }\end{array}$ \\
\hline \multirow{2}{*}{ Age (in Years) } & Present & \multicolumn{2}{|c|}{$35.87 \pm 7.05$} & $-2.268^{*}$ \\
\hline & Absent & \multicolumn{2}{|c|}{$41.31 \pm 7.80$} & $\mathrm{P}<0.05$ \\
\hline \multirow{6}{*}{ Marital status } & \multirow{3}{*}{ Present } & Single & $10(21.30 \%)$ & \multirow{6}{*}{$\begin{array}{l}5.01 * * \\
\text { ntegr }\end{array}$} \\
\hline & & Married & $33(70.20 \%)$ & \\
\hline & & Separated & $4(8.50 \%)$ & \\
\hline & \multirow{3}{*}{ Absent } & Single & $0(0.00 \%)$ & \\
\hline & & Married & $13(100.00 \%)$ & \\
\hline & & Separated & $0(0.00 \%)$ & \\
\hline \multirow{8}{*}{ Education } & \multirow{4}{*}{ Present } & Nil & $2(4.30 \%)$ & \multirow{8}{*}{$4.475^{* * *}$} \\
\hline & & Primary & $12(25.50 \%)$ & \\
\hline & & Secondary & $\begin{array}{l}15(31.90 \%) \\
\end{array}$ & \\
\hline & & Graduate & $18(38.30 \%)$ & \\
\hline & \multirow{4}{*}{ Absent } & Nil & $1(7.70 \%)$ & \\
\hline & & Primary & $7(53.80 \%)$ & \\
\hline & & Secondary & $2(15.40 \%)$ & \\
\hline & & Graduate & $3(23.10 \%)$ & \\
\hline \multirow{12}{*}{ Occupation } & \multirow{6}{*}{ Present } & Agriculture & $8(17.00 \%)$ & \multirow{12}{*}{9.61} \\
\hline & & Labourer & $10(21.30 \%)$ & \\
\hline & & Business & $15(31.90 \%)$ & \\
\hline & & Service & $1(2.10 \%)$ & \\
\hline & & Professional & $12925.50 \%)$ & \\
\hline & & Not Employed & $1(2.10 \%)$ & \\
\hline & \multirow{6}{*}{ Absent } & Agriculture & $7(53.80 \%)$ & \\
\hline & & Labourer & $2(15.40 \%)$ & \\
\hline & & Business & $1(7.70 \%)$ & \\
\hline & & Service & $1(7.70 \%)$ & \\
\hline & & Professional & $2(15.40 \%)$ & \\
\hline & & Not Employed & $0(0.00 \%)$ & \\
\hline \multirow{4}{*}{ Family Type } & \multirow{2}{*}{ Present } & Nuclear & $33(70.20 \%)$ & \multirow{4}{*}{$1.080^{*}$} \\
\hline & & Extended & $14(29.80 \%)$ & \\
\hline & \multirow{2}{*}{ Absent } & Nuclear & $11(84.60 \%)$ & \\
\hline & & Extended & $2(15.40 \%)$ & \\
\hline
\end{tabular}

Level of Significance: ${ }^{*}-p<0.05,{ }^{* *}-p<0.01,{ }^{* * *}{ }_{-}<0.001$, NS-Not Significant

Association between High Risk Behaviour as determined by Event-analysis and socio-demographic variables. The mean age of individuals with high risk behaviour was 35.87 years. Majority of the individuals were married. High Risk Behaviour was high among graduates followed by individuals with secondary education also was noted to be more among the individuals who had business as their occupation followed by professionals. Majority of the individuals with High Risk Behaviour belonged to a nuclear family.
Table 6: Association of High Risk Behaviour with family history and severity of dependence.

\begin{tabular}{|c|c|c|c|c|}
\hline Variable & $\begin{array}{l}\text { High Risk } \\
\text { Behaviour }\end{array}$ & \multicolumn{2}{|c|}{ Mean \pm SD/Frequency (\%) } & $\begin{array}{c}\text { t-value/Chi } \\
\text { square }\end{array}$ \\
\hline \multirow{4}{*}{$\begin{array}{l}\text { Family } \\
\text { History }\end{array}$} & \multirow{2}{*}{ Present } & Nil & 8 (17.00\%) & \multirow{4}{*}{$5.9 * \mathrm{P}<0.05$} \\
\hline & & Present & 39 (83.00\%) & \\
\hline & \multirow{2}{*}{ Absent } & $\mathrm{Nil}$ & 10 (76.90\%) & \\
\hline & & Present & $3(23.10)$ & \\
\hline \multirow{6}{*}{$\begin{array}{l}\text { SAD Q } \\
\text { Severity }\end{array}$} & \multirow{3}{*}{ Present } & Mild & $1(2.10 \%)$ & \multirow{6}{*}{$5.051 * *$} \\
\hline & & Moderate & $19(40.40 \%)$ & \\
\hline & & Severe & $27(57.40 \%)$ & \\
\hline & \multirow{3}{*}{ Absent } & Mild & $3(23.10 \%)$ & \\
\hline & & Moderate & 9 (69.20\%) & \\
\hline & & Severe & $1(7.70 \%)$ & \\
\hline
\end{tabular}

Level of Significance: ${ }^{*} p<0.05, * * p<0.01, * * *<0.001$, NS-Not Significant

High Risk Behaviour was found to be much more in individuals with family history of dependence. Results also reveal the significant correlation between severity of dependence and High Risk Behaviour. High Risk Behaviour was found to be significantly high in individuals with severe dependence when compared to that of mild and moderate dependent individuals.

Table 7: Association between High Risk Behaviour and sensation seeking behavior.

\begin{tabular}{|c|c|c|c|}
\hline VARIABLES & $\begin{array}{l}\text { High Risk } \\
\text { Behaviour }\end{array}$ & MEAN $\pm S D$ & t- value * \\
\hline \multirow{2}{*}{$\begin{array}{c}\text { Thrill \& adventure Seeking (subscale of } \\
\text { Sensation Seeking Scale) }\end{array}$} & Present & $5.21 \pm 0.72$ & \multirow{2}{*}{$5.795^{*}$} \\
\hline & Absent & $4.0 \pm 0.41$ & \\
\hline \multirow{2}{*}{$\begin{array}{l}\text { Experience Seeking (subscale of } \\
\text { Sensation Seeking Scale) }\end{array}$} & Present & $4.3 \pm 0.6$ & \multirow{2}{*}{$4.835^{*}$} \\
\hline & Absent & $3.3 \pm 0.6$ & \\
\hline \multirow{2}{*}{$\begin{array}{c}\text { Disinhibition (subscale of Sensation } \\
\text { Seeking Scale) }\end{array}$} & Present & $5.9 \pm 0.8$ & \multirow{2}{*}{$7.958^{*}$} \\
\hline & Absent & $4.0 \pm 0.6$ & \\
\hline \multirow{2}{*}{$\begin{array}{l}\text { Boredom Susceptibility (subscale of } \\
\text { Sensation Seeking Scale) }\end{array}$} & Present & $5.5 \pm 0.6$ & \multirow{2}{*}{$7.069 *$} \\
\hline & Absent & $4.2 \pm 0.6$ & \\
\hline \multirow{2}{*}{ Total Sensation Seeking Scale Score } & Present & $20.9 \pm 2.1$ & \multirow{2}{*}{$8.761 *$} \\
\hline & Absent & $15.5 \pm 1.4$ & \\
\hline
\end{tabular}

Level of significance: ${ }^{*}-\mathrm{p}<0.05,{ }^{* *}$-p $<0.01,{ }^{* * *}$-p $<0.001$, NS-Not significant

Thrill and adventure seeking, experience seeking, disinhibition and boredom susceptibility were found to be significantly high in individuals with High Risk Behaviour.

Table 8: Association between High Risk Behaviour and impulsivity.

\begin{tabular}{|c|c|c|c|}
\hline VARIABLES & $\begin{array}{c}\text { High Risk } \\
\text { Behaviour }\end{array}$ & MEAN \pm SD & t- value * \\
\hline \multirow{2}{*}{$\begin{array}{c}\text { MOTOR ( subscale of Barratt's } \\
\text { Impulsiveness Scale) }\end{array}$} & present & $30.1 \pm 1.9$ & \multirow{2}{*}{$10.791^{*}$} \\
\cline { 2 - 3 } & Absent & $24.8 \pm 1.6$ & \\
\hline $\begin{array}{c}\text { NONPLANNING (subscale of } \\
\text { Barratt's Impulsiveness Scale) }\end{array}$ & Present & $31.3 \pm 1.8$ & \multirow{2}{*}{$10.129^{*}$} \\
\cline { 2 - 3 } $\begin{array}{c}\text { COGNITIVE (subscale of Barratt's } \\
\text { Impulsiveness Scale) }\end{array}$ & Absent & $25.6 \pm 1.7$ & \\
\cline { 2 - 3 } & Present & $23.1 \pm 1.5$ & \multirow{2}{*}{$6.378^{*}$} \\
\hline \multirow{2}{*}{ BIS (Total Score) } & Present & $20.0 \pm 1.7$ & \\
\cline { 2 - 3 } & Absent & $70.4 \pm 4.5$ & \multirow{2}{*}{$10.824^{*}$} \\
\hline
\end{tabular}

Level of significance: ${ }^{*}-p<0.05,{ }^{* *}-p<0.01,{ }^{* * *}-p<0.001$, NS-Not significant. 
Individuals with High Risk Behaviour scored significantly high on all domains of impulsivity scale.

Table 9: Association between Family history and High Risk Behaviour.

\begin{tabular}{|c|c|c|c|}
\hline Variables & Family h/o & $\begin{array}{c}\text { Mean } \pm \text { SD/ Frequency } \\
(\%)\end{array}$ & $\begin{array}{l}\text { t-value / Chi } \\
\text { square/ F- } \\
\text { Value }\end{array}$ \\
\hline \multirow{4}{*}{ High Risk Behaviour } & Nil & PRESENT - $8(44.4 \%)$ & \multirow{4}{*}{$17.400^{*}$} \\
\hline & & ABSENT - $10(55.6 \%)$ & \\
\hline & Present & PRESENT - 39 (92.9\%) & \\
\hline & & ABSENT - 3 (7.1\%) & \\
\hline \multirow{4}{*}{ RTA } & Nit & PRESENT - 7 (38.9\%) & \multirow{4}{*}{2.156} \\
\hline & IVII & ABSENT - 11 (61.1\%) & \\
\hline & Present & PRESENT - 25 (59.5\%) & \\
\hline & & ABSENT - $17(40.5 \%)$ & \\
\hline \multirow{4}{*}{ CRIME \& VIOLENCE } & Nit & PRESENT - 1 (5.6\%) & \multirow{4}{*}{$4.543^{*}$} \\
\hline & IVII & ABSENT - $17(94.4 \%)$ & \\
\hline & Procit & PRESENT - 13 (31.0\%) & \\
\hline & present & ABSENT - $29(69.0 \%)$ & \\
\hline \multirow{4}{*}{ SELF INJ. BEHAVIOUR } & Nit & PRESENT - $3(16.7 \%)$ & \multirow{4}{*}{$0.639 \mathrm{~s}$} \\
\hline & IVII & ABSENT - 15 (83.3\%) & \\
\hline & Drescot & PRESENT - 11 (26.2\%) & \\
\hline & Present & ABSENT - $31(73.8 \%)$ & \\
\hline \multirow{4}{*}{$\begin{array}{l}\text { HIGH RISK SEXUAL } \\
\text { BEHAVIOUR }\end{array}$} & Nit & PRESENT - $5(27.8 \%)$ & \multirow{4}{*}{$8.739 *$} \\
\hline & & ABSENT - $13(72.2 \%)$ & \\
\hline & Present & PRESENT - $29(69.0 \%)$ & \\
\hline & Present & ABSENT - $13(31.0 \%)$ & \\
\hline
\end{tabular}

Level of significance: $*-p<0.05, * *-p<0.01, * * *-p<0.001$, NS-Not significant.

Crime and Violence and High Risk Sexual Behaviour were found to be significantly high in individuals with family history of dependence.

Table 10: Association between Family history and Sensation Seeking Behaviour.

\begin{tabular}{|c|c|c|c|}
\hline \multirow{2}{*}{ Variables } & Family H/O & $\begin{array}{c}\text { Mean } \pm \text { SD/ } \\
\text { Frequency ( \% ) }\end{array}$ & $\begin{array}{c}\text { t-value / Chi } \\
\text { square / F- } \\
\text { Value }\end{array}$ \\
\hline \multirow{2}{*}{ TAS } & Nil & $4.50 \pm 0.9$ & \multirow{2}{*}{$-2.911^{*}$} \\
\cline { 2 - 3 } & Present & $5.14 \pm 0.8$ & \\
\hline \multirow{2}{*}{ ES } & Nil & $3.83 \pm 0.8$ & \multirow{2}{*}{-1.537} \\
\cline { 2 - 3 } & Present & $4.14 \pm 0.7$ & \\
\hline \multirow{2}{*}{ BS } & Nil & $4.94 \pm 0.8$ & \multirow{2}{*}{$-1.999^{*}$} \\
\cline { 2 - 3 } DIS & Present & $5.38 \pm 0.8$ & \multirow{2}{*}{$-3.493^{*}$} \\
\cline { 2 - 3 } & Nil & $4.78 \pm 1.1$ & \multirow{2}{*}{$-3.045^{*}$} \\
\hline \multirow{2}{*}{$\begin{array}{c}\text { SENSATION SEEKING } \\
\text { TOTAL SCORE }\end{array}$} & Nil & $18.74 \pm 0.9$ & \\
\cline { 2 - 3 } & Present & $20.40 \pm 2.7$ & \\
\hline
\end{tabular}

Level of significance: ${ }^{*}$-p $<0.05,{ }^{* *}$-p $<0.01,{ }^{* * *}$-p $<0.001$, NS-Not significant.

Thrill and adventure seeking behaviour, boredom susceptibility and disinhibition were found to be significantly associated with family history of dependence. Sensation seeking behaviour has a positive correlation with family history of dependence.

Int J Intg Med Sci 2018;5(7):678-86. ISSN 2394 - 4137
Table 11: Association between Family history and impulsivity.

\begin{tabular}{|c|c|c|c|}
\hline Variables & Family (H/O) & $\begin{array}{c}\text { Mean } \pm \text { SD/ } \\
\text { Frequency ( \%) }\end{array}$ & $\begin{array}{c}\text { t-value / Chi } \\
\text { square / F-Value }\end{array}$ \\
\hline \multirow{2}{*}{ MOTOR } & Nil & $27.00 \pm 3.1$ & \multirow{2}{*}{$-4.505 *$} \\
\hline & Present & $30.74 \pm 2.4$ & \\
\hline \multirow{2}{*}{ NON-PLANNING } & Nil & $27.56 \pm 3.2$ & \multirow{2}{*}{$-4.432^{*}$} \\
\hline & Present & $31.17 \pm 2.1$ & \\
\hline \multirow{2}{*}{ COGNITIVE } & Nil & $21.22 \pm 2.5$ & \multirow{2}{*}{$-2.787^{*}$} \\
\hline & Present & $23.00 \pm 1.5$ & \\
\hline \multirow{2}{*}{ BIS-TOTAL SCORE } & Nil & $75.78 \pm 8.4$ & \multirow{2}{*}{$-4.235^{*}$} \\
\hline & Present & $84.90 \pm 5.4$ & \\
\hline
\end{tabular}

Level of significance: ${ }^{*}$-p $<0.05,{ }^{* *}-p<0.01, * * *$-p $<0.001$, NS-Not significant

Individuals with family history of dependence scored high on all domains of impulsivity scores when compared to others.

\section{DISCUSSION}

The aim of the study was to assess the prevalence of high risk behaviour and its association with personality constructs such as sensation seeking and impulsivity in male patients with a diagnosis of ADS. The sample comprised of 60 male patients admitted as in-patients in Narayana general hospital. The mean age of the sample was $37.05 \pm 7.4$ years with $76.7 \%$ of them being married followed by $16.7 \%$ individuals were single. Majority of the individuals belonged to a nuclear family (73.7\%) followed by individuals belonging to an extended family (26.3\%). Among the individuals recruited for the study $35 \%$ were graduates followed by $37.5 \%$ who only had primary education.70\% of the individuals recruited for the study have family history of alcohol dependence. Severity of dependence shows significant association with family history (odds ratio 1.52).

The results of the current study indicated significant prevalence of high risk behaviour in patients with alcohol dependence. High risk sexual behaviour (56.7\%) was the most common high risk behaviour observed followed by road traffic accident (53.3\%). Crime and violence and self-injurious behaviour were present in about $20 \%$ of the individuals. The results of the current study were consistent with the findings of Biju Poulose et al., [14] the most common high risk behaviour was road traffic accident followed by high risk sexual behaviour. In a national survey by Temple MT et.al., [15] findings suggest that the relationship between 
drinking and risky sex is the result of a complex interaction among personality, situational, and behavioural factors.

Personality constructs contributing to the high risk behaviour sensation seeking and impulsivity were evaluated. Patients with high risk sexual behaviour scored significantly in the disinhibition domain of sensation seeking. The results of the current study revealed that high risk sexual behaviour was much more in young adults when compared to that of the older age group which is consistent with the study conducted by Dermen KH et.al., [16] about Sex-related alcohol expectancies as moderators of the relationship between alcohol use and risky sex in adolescents found that alcohol use was associated with greater risk taking primarily among respondents who expected alcohol to increase risky sexual behaviour. Justus AN et.al., [17] studied the influence of traits of disinhibition on the association between alcohol use and risky sexual behaviour. There was a significant correlation between alcohol use and risky sexual behaviour when considered alone. Association between alcohol use and risky sexual behaviour is best explained by their common association with excitement seeking alone. However, when controlling for the effects of personality, the correlation was insignificant. Both excitement seeking and social deviance proneness were found to be significantly associated with alcohol use, and excitement seeking was significantly related to risky sexual behaviour. The current study also showed significant correlation between high risk behaviour and personality constructs of sensation seeking scale such as disinhibition and thrill and adventure seeking.

In a study by Laphamet al et.al., [18] the prevalence of psychiatric disorders rates showed that $70 \%$ of the male offenders had a diagnosis of Alcohol Dependence Syndrome, whereas only $35.7 \%$ of the men had a diagnosis of dependence syndrome in the National Co-morbidity Sample. Fish et al et.al., [19] and McCord et.al., [20] found that in individuals convicted with drunk driving nearly 50\% were Alcohol Dependent at follow-up, with a constant increase in the prevalence of a diagnosis of Alcohol Dependence Syndrome in following convictions as seen by $70 \%$ of the second- time offenders, with further increases to nearly all those convicted with multiple convictions for drunken driving fulfilling the criteria for dependence. Thus, what these studies show are that Road Traffic Accidents are one of the most common high-risk behaviour seen in people with Alcohol Dependence Syndrome as has also been noticed in our study but only second to high risk sexual behaviour which may be attributed to rural background of the recruited individuals were road traffic accidents are known to be low. James B et.al, [21] conducted a meta-analysis to investigate the association between sensation seeking and alcohol use. On analysis of the four sensation-seeking components indicated that disinhibition was most strongly correlated with alcohol use. The current study also reveals a strong correlation between severity of alcohol and sensation seeking predominantly with disinhibition component of the scale followed by thrill and adventure seeking.

Moeller et.al., [22] in their study concluded that impulsivity is a risk factor and a consequence of substance abuse which has to be addressed during both the initiation and maintenance of substance abuse treatment. The current study revealed that the prevalence of impulsive personality traits were much higher in people with high risk behaviour and substance use suggesting a strong correlation between all the three.

In the current study severity of dependence was much higher among young adults and there was a significant correlation with high risk behaviour and sensation seeking. Scores on the impulsivity scale were also much higher for the young adults. The findings of this study are consistent with the results of a study conducted by Dom et.al., [23] found that the symptom severity of the early-onset alcoholics alcoholuse disorder and related problems was higher than that of the late-onset alcoholics. Furthermore, the early-onset alcoholics had higher levels of impulsivity, sensation seeking, and aggression relative to the late-onset alcoholics. The differences in impulsivity remained after an analysis controlling for the effect of aggressiveness. It was found that cigarette smoking was positively correlated with impulsiveness across 
alcoholic subgroups.

Antonio Verdejo-García et.al., [24] in their study on negative emotion-driven impulsivity as a predictor of substance dependence problems stated that impulsivity is predominant among users of several drugs of abuse including alcohol, cocaine, and amphetamines, and it is considered a risk factor for later development of alcohol and substance abuse and dependence among the different impulsivity dimensions, urgency was the best predictor of severity of medical, employment, alcohol, drug, family/ social, legal and psychiatric problems in individuals with substance dependence. Urgency is characterized by a tendency to act impulsively in response to negative emotional states and was found in $83 \%$ of the individuals with substance dependence. Negative emotion states were not evaluated in the present study. On the impulsivity scale young adults had higher scores in non-planning component followed by motor component whereas the individuals belonging to higher age group had less impulsivity and their scores were high on cognitive component of the BIS. From the above studies it can concluded that high risk behaviour is predominant among individuals with substance dependence and various personality constructs such as sensation seeking and impulsivity contribute towards their behaviour. A comprehensive management of individuals with substance dependence needs to address these issues to reduce the relapse rates which are high among this population.

\section{CONCLUSION}

The present study aimed to find out the association between High Risk Behaviour and Alcohol Dependence Syndrome. There was a very high occurrence of high-risk behavior in alcoholics, risky sexual behavior was the commonest followed by road traffic accidents. Severity of alcohol dependence was significantly associated with the occurrence of high-risk behaviour. Patients scoring high on certain personality constructs such as sensation seeking and impulsivity were particularly vulnerable to indulge in high-risk behaviour. Analysis indicated that severity of alcohol dependence interacted with personality variables and demographic characteristics (younger age, married, primary education) resulting in occurrence of high- risk behavior. The results of the present study suggest that treatment interventions must inquire into and specifically target such high-risk behaviour to reduce morbidity and mortality associated with severe alcohol dependence.

\section{REFERENCES}

[1]. Keller M and Vaillant GE (2010): Alcohol consumption. Encyclopædia Britannica [online] Available from: http://www.britannica.com/EBchecked/topic/ 13398/alcohol-consumption [Accessed 28 April 2010]

[2]. NHMRC (2009): Australian guidelines To Reduce Health Risks from Drinking Alcohol. National Health and Medical Research Council, Commonwealth of Australia.

[3]. White IR. The Level of Alcohol Consumption at Which All-Cause Mortality Is Least. Journal of Clinical Epidemiology. 1999;52(10):967-75

[4]. Harwood HJ, Fountain D and Livermore G (1998): Economic Costs of Alcohol Abuse and Alcoholism. Recent Developments in Alcoholism (book series), Volume 14

[5]. Murray, C.J.L., and Lopez, A.D. (Eds). The Global Burden of Disease. A comprehensive assessment of mortality and disability from diseases, injuries and risk factors in 1990 and projected to 2020. Cambridge MA. Harvard School of Public Health, on behalf of World Health Organization and the World Bank. (Global Burden of Disease and Injury Series, vol1). 1996.

[6]. Ponnudurai R., Jayakar J., Raju B., Pattumuthu. An epidemiological Study of Alcoholism. Indian Journal of Psychiatry. 1991;33:176-9

[7]. Leigh, B.C. The risks of drinking among young adults. Peril, chance, adventure: concepts of risk, alcohol use and risky behaviour in young adults. Addiction. 1999;94 (3):371-83.

[8]. Wood, P.B., Cochran, J.K., Pfefferbaum, B., Arneklau, B.J. Sensation-seeking and delinquent substance use: An extension of the learning theory. Journal of Drug Issues 1995;25: 173-93.

[9]. Uckermann, M. Sensation- seeking: the balance between risk and reward, in: Lipsitt, L.P. and Mitnick, L.L. ( Eds). Self Regulatory Behaviour and Risk Taking: cause and consequences, 143-152. (Norwood, NJ, Ablex).

[10]. The World Health Report - 2003, The World Health Organization.

[11]. Stockwell, T., Murphy, D., Hodgson, R. The severity of alcohol dependence questionnaire: Its use, reliability and validity. British Journal of Addiction. 1983;78(2):45-156..

[12]. Basu, D., Verma, V.K., Malhotra, S., Malhotra, A. Sensation seeking scale: Indian Adaptation. Indian Journal of Psychiatry 1993;35(3):155-8. 
[13].Barratt, E.S., Stanford, M.S. Impulsiveness, in the personality characteristics of the Personality Disordered client. Edited by Costello, C.G. New York. Wiley, 1995;91-118.

[14]. Biju Poulose, Krishnamachari Srinivasan. High risk behaviours following alcohol use in alcohol dependent men. Indian J Med Res 2009;129:376-81.

[15]. Temple MT, Leigh BC, Schafer J. Unsafe sexual behavior and alcohol use at the event level: results of a national survey. J Acquir Immune Defic Syndr. 1993;6(4):393-401.

[16]. Dermen KH, Cooper ML, Agocha V B. Sex-related alcohol expectancies as moderators of the relationship between alcohol use and risky sex in adolescents. J Stud Alcohol. 1998;59(1):71-7.

[17]. Justus AN, Finn PR, Steinmetz JE. The influence of traits of disinhibition on the association between alcohol use and risky sexual behavior. Alcohol Clin Exp Res. 2000;24(7):1028-35.

[18]. Lapham SC, Smith E, Cde Baca J, Chang I, Skipper BJ, Baum G, et.sal. Prevalence of psychiatric disorders among persons convicted with driving while impaired. Archives of General Psychiatry 2001;58:943-949.
[19]. Fine, E.W., Scoles, P., Mulligan, M. Under the influence: characteristics and drinking practices of persons arrested for the first time for drunk driving, with treatment implications. Public Health Report. 1975;90:424-9.

[20]. McCord, J. Drunken drivers in longitudinal perspective. Journal of Studies on Alcohol 1984;45:316-20.

[21]. James B. Hittner, Rhonda Swickert. Sensation seeking and alcohol use: A meta-analytic review. Addictive Behaviours. 2006;31( 8):1383-1401.

[22]. Moeller, F. Gerard MD; Dougherty, Donald M. Impulsivity and Substance Abuse: What Is the Connection? Addictive Disorders \& Their Treatment. 2002;1(1):3-10.

[23]. Dom. G, W. Hulstijn, B. Sabbe. Differences in impulsivity and sensation seeking between early- and late-onset alcoholics. Addictive Behaviors. 2006;31(2): 298-308.

[24]. Antonio Verdejo-Garcíaa, , , Antoine Becharab, c, Emily C. Recknord, Miguel Pérez-Garcíae.Negative emotion-driven impulsivity predicts substance dependence problems. Drug and Alcohol Dependence. 2007;91(2-3): 213-9.

How to cite this article: Chandra Sekhar A, Eshwar Reddy Ravula. ASSOCIATION BETWEEN HIGH RISK BEHAVIOUR AND ALCOHOL DEPENDENCE SYNDROME. Int J Intg Med Sci2018;5(7):678-686. DOI: 10.16965/ijims.2018.123 